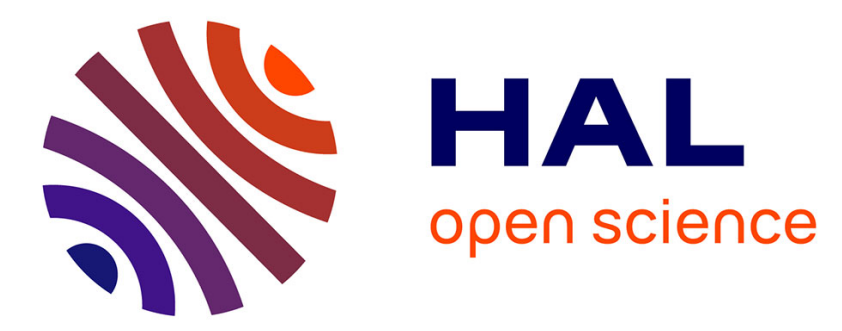

\title{
Tracking the Frenet-Serret frame associated to a highly maneuvering target in $3 \mathrm{D}$
}

Marion Pilté, Silvère Bonnabel, Frédéric Barbaresco

\section{To cite this version:}

Marion Pilté, Silvère Bonnabel, Frédéric Barbaresco. Tracking the Frenet-Serret frame associated to a highly maneuvering target in 3D. 2017. hal-01570709

\section{HAL Id: hal-01570709 \\ https:/ /hal-mines-paristech.archives-ouvertes.fr/hal-01570709}

Preprint submitted on 31 Jul 2017

HAL is a multi-disciplinary open access archive for the deposit and dissemination of scientific research documents, whether they are published or not. The documents may come from teaching and research institutions in France or abroad, or from public or private research centers.
L'archive ouverte pluridisciplinaire HAL, est destinée au dépôt et à la diffusion de documents scientifiques de niveau recherche, publiés ou non, émanant des établissements d'enseignement et de recherche français ou étrangers, des laboratoires publics ou privés. 


\title{
Tracking the Frenet-Serret frame associated to a highly maneuvering target in 3D
}

\author{
Marion Pilté, Silvère Bonnabel, Frédéric Barbaresco
}

\begin{abstract}
In this paper we consider the problem of tracking a maneuvering aircraft. The dynamical model used is based on assumptions of nearly constant tangential velocity, curvature, and torsion, of the target trajectory. Using the Frenet-Serret frame, that is, an element of the special orthogonal group $\operatorname{SO}(3)$ we cast the tracking problem into a filtering on Lie groups framework. We then use an invariant extended Kalman filter to estimate the various quantities involved. The resulting filter is simple to implement, and shown to gracefully accomodate some realistic target trajectories.
\end{abstract}

\section{INTRODUCTION}

In radar applications, track maintenance is one essential component of the process. Mathematically, it boils down to a filtering problem, where one must filter the current position of the aircraft as well as its velocity and possibly higher order derivatives, from noisy position measurements. We will refer to this problem simply as "target tracking". When the aircraft is maneuvering, the problem is difficult due to the unpredictable nature of the motion. This area has been the object of extensive research over the four past decades, see [1]. The main degrees of freedom for tracking are 1the dynamical model describing the motion of the target, and 2- the (statistical) filter used. As concerns dynamical models, there are many possibilities, but linear models are often used in industrial applications, the most famous being the Singer model [2]. As concerns the filters, a simple robust solution is the noise process-adaptative Kalman filter of Castella [3]. More modern approaches include particle filters [4] and the reference filter for tracking which is the interacting multiple model (IMM) filter, see e.g. [1]. The latter filter runs banks of (extended) Kalman filters in parrallel based on various models and assess weights to each model by evaluating likelihood of the measured outputs. This allows accomodating the various types of targets and degrees of maneuverability a single radar can be confronted with. The acadamic community has now largely turned to the challenges of multi-target tracking, with joint applications in video, see [5].

Nowadays, the radar air defense industry is facing novel challenges with ever increasingly maneuvering targets. Some targets can reach Mach 7 velocities with $15 g$ accelerations. A way to inject some structure through a motion model

\footnotetext{
M. Pilté is with Thales Air System and Mines ParisTech, PSL Research University, Center for Robotics, marion.pilte@mines-paristech. fr

S. Bonnabel is with Mines ParisTech, PSL Research University, Center for Robotics, silvere.bonnabel@mines-paristech. fr

F. Barbaresco is with Thales Air System, frederic.barbarescodthalesgroup.com
}

into a trajectory that is deliberately trying to make the radar lose track of it, is to resort to physical considerations: The changes in aerodynamic lift and thrust-drag accelerations are limited, and those accelerations can in fact be expected to be piecewise nearly constant.

In the present paper, we propose to study the problem of tracking a highly maneuvering target in the 3D space. We propose as a very simple geometric model to use the FrenetSerret formulas to describe the motion and assuming nearly constant curvature and torsion. This model includes helical motions that are particularly challenging to track. Our model can be related to [6], [7] and more recently [8], see Section II-B for more information. The position $x$ is a 3D vector and the Frenet-Serret frame a rotation matrix $R$ of $S O(3)$, so $(x, R)$ can be viewed as an element of the Lie group $S E(3)$. Using this fact, we cast the tracking problem (partially) into a filtering problem on Lie groups. Slightly extending the Invariant Extended Kalman Filter (IEKF), introduced in [9] and [10], we obtain a novel tracking algorithm. The IEKF is indeed a recent methodology that accounts for the geometric nature of the state space, and comes with convergence properties [11]. It can also be related to the discrete EKF on Lie groups of [12], or to the generalized multiplicative EKF of [13], see also [14].

The paper is organized as follows. In Section II we derive a stochastic evolution model from the Frenet-Serret equations and discuss the design of estimation algorithms. In Section III, we explain how the theory of [11] can be adapted when the state space consists of a Lie group and of additional quantities, and we describe the IEKF equations for the tracking problem. Finally, in Section IV, simulations illustrate the relevance of the filter.

\section{PROBLEM AND PROPOSED MODELING}

Consider a vehicle, say, an aircraft, evolving in the $3 \mathrm{D}$ space. It seems natural to describe the motion using piecewise constant control commands in a frame attached to the aircraft, that is, using intrinsic coordinates, as proposed in e.g., [15]. As rotations of the aircraft around its velocity vector are unobservable from position measurements only, we propose to model its trajectory using Frenet-Serret formulas and to assume the tangential velocity, curvature, and torsion to be nearly constant. Denoting by $x_{t} \in \mathbb{R}^{3}$ the position of the target, the Frenet-Serret formulas write

$$
\begin{aligned}
& \frac{d}{d t} x_{t}=u T \\
& \frac{d}{d t} T=u \kappa N, \frac{d}{d t} N=u(-\kappa T+\tilde{\tau} B), \frac{d}{d t} B=-u \tilde{\tau} N
\end{aligned}
$$


where $u \geq 0$ will be referred to as the (norm of the) tangential velocity, $\kappa$ is the curvature, $\tilde{\tau}$ is the torsion, and $T, N, B$ are all unit vectors perpendicular to each other. Those equations are also known as Frenet-Serret theorem and using a concise matrix notation, equation (2) can be re-written

$$
\frac{d}{d t}\left(\begin{array}{lll}
T & N & B
\end{array}\right)=u\left(\begin{array}{lll}
T & N & B
\end{array}\right)\left(\begin{array}{ccc}
0 & -\kappa & 0 \\
\kappa & 0 & -\tilde{\tau} \\
0 & \tilde{\tau} & 0
\end{array}\right)
$$

Defining $\gamma$ and $\tau$ as $\gamma=u \kappa, \tau=u \tilde{\tau}$ we finally get:

$$
\frac{d}{d t}\left(\begin{array}{lll}
T & N & B
\end{array}\right)=\left(\begin{array}{lll}
T & N & B
\end{array}\right)\left(\begin{array}{ccc}
0 & -\gamma & 0 \\
\gamma & 0 & -\tau \\
0 & \tau & 0
\end{array}\right)
$$

In the sequel, the parameters $\gamma$ and $\tau$ will be referred to as curvature and torsion with a slight abuse of language, but in the application section, we will come back to $\kappa$ and $\tilde{\tau}$.

\section{A. Proposed model for the target}

Using Frenet-Serret formulas, we let $R_{t} \in S O(3)$ be the matrix $R_{t}=\left(\begin{array}{lll}T & N & B\end{array}\right)$. We then assume nearly constant tangential velocity, curvature, and torsion, leading to the following model for the dynamics:

$$
\begin{gathered}
\frac{d}{d t} x_{t}=R_{t} v_{t}+w_{t}^{x}, \quad \frac{d}{d t} R_{t}=R_{t}\left(\omega_{t}+w_{t}^{\omega}\right)_{\times}, \\
\frac{d}{d t} \gamma_{t}=w_{t}^{\gamma}, \quad \frac{d}{d t} \tau_{t}=w_{t}^{\tau}, \quad \frac{d}{d t} v_{t}=w_{t}^{u}
\end{gathered}
$$

where the curvature $\gamma_{t}$, the torsion $\tau_{t}$ and velocity norm $u_{t}$ are unknown parameters, $\omega_{t}=\left(\begin{array}{lll}\tau_{t} & 0 & \gamma_{t}\end{array}\right)^{T}, v_{t}=\left(\begin{array}{lll}u_{t} & 0 & 0\end{array}\right)^{T}$, and $w_{t}^{\omega}, w_{t}^{x}, w_{t}^{\gamma}, w_{t}^{\tau}, w_{t}^{u}$ are white noises that account for small changes over time in the motion parameters. Moreover, we let $(a)_{\times} \in \mathbb{R}^{3 \times 3}$ denote the skew symmetric matrix associated with cross product with the vector $a \in \mathbb{R}^{3}$.

Remark 1: The model (5) can be easily extended to accommodate nearly constant accelerations of the Singer model type [2], by replacing $\frac{d}{d t} v_{t}=w_{t}^{u}$ with $\frac{d}{d t} v_{t}=a_{t}$ and $\frac{d}{d t} a_{t}=-\alpha a_{t}+w_{t}^{u}$. The filter below can easily be adapted.

The observations are the noisy positions at times $t_{1}, t_{2}, \ldots$ :

$$
Y_{n}=x_{t_{n}}+V_{n}
$$

where the $V_{n}$ 's are Gaussian independent observation noises in $\mathbb{R}^{3}$. It is also common to express the radar position measurements in spherical coordinates of range, elevation and azimuth angles which leads to a nonlinear measurement model. Even if it poses additional difficulties in its own right, it is not the main object of the present paper and the technique presented here can be adapted linearizing the output map as in the EKF methodology. There are also techniques to transform (more precisely estimate without bias) the measurements from spherical to Cartesian, see [16].

\section{B. Associated difficulties and links with prior literature}

The proposed model (5) seems well suited to the tracking of highly maneuvering targets. Indeed it is closely related to constant commands in the body frame, albeit different. Devising a filter to track equations (1)-(2), or more precisely the retained model (5), from position measurements is difficult for various reasons. First, because there are many quantities to be estimated only from position measurements and which appear nonlinearly in the equations. Then, because of the constraint of $T, N, B$ forming an orthonormal base of the 3D space. It is not trivial to encode such nonlinear constraints in an extended Kalman filter. Using the model (5), we see that the pair $\left(x_{t}, R_{t}\right)$ defines an element of the Lie group $S E(3)$. This indicates (part of) the state could be estimated by bringing to bear the Lie group structure underlying the state space. There has been extensive work on observers on Lie groups over the past years. However, for target tracking applications it is important that the filter output a covariance matrix, preventing the use of deterministic observers. Indeed, radar tracking involves the non trivial task of associating radar reports (so called "plots") with targets. This task (which is not considered in the present paper that is only concerned with tracking) is critical and requires an associate window based on the estimation error dispersion (covariance). This is why we resort to a Lie group based extended Kalman filter - the invariant EKF of [11] - for the tracking task.

There has been various prior attempts to use intrinsic coordinates to describe the target motion. The most prominent work in this direction is, to our best knowledge, the pioneering work of Antoulas and Bishop, see e.g., [7], [17]. If we assume the acceleration of the target is large with respect to the gravity vector field $g$, which is the case for highly maneuvering targets the NCS-CT model of [7] writes

$$
\dddot{x}_{t}=\frac{\dot{x}_{t} \times \ddot{x}_{t}}{\left\|\dot{x}_{t}\right\|^{2}} \times \ddot{x}_{t}
$$

This equation is obtained assuming the kinematic acceleration $\ddot{x}_{t}$, when projected onto the body frame, is constant. Although the motivations for this model is akin to our motivations, the obtained equations are slightly different. Note indeed that, first the velocity of the target $\dot{x}_{t}$ must always be different from zero for the model to be valid, which is not the case with equations (5). Moreover, the model (7) is based on a zero torsion assumption. As a result quoting [17] "necessary condition for the model to be an accurate representation of actual trajectories is for the real trajectories to have small torsions". It is easily seen that the retained model (7) does not lend itself very well to extended Kalman filtering due to its strong nonlinearities. The authors thus propose a geometric filter, which is in fact a deterministic observer (of the Luenberger type [18]), but for a nonlinear model, see [19].

Another work that is akin to the proposed Frenet-Serret model (1)-(2) or equivalently (5) in state space form, is [8]. The proposed model is based on constant tangential and normal accelerations and constant-plane motion, and differs from [20], [1] only in the way the noise enters the system. The authors assume indeed the system to be deterministic, and the acceleration to jump from time to time. They then use a particle filter to select the most likely accelerations. This method is fundamentally different from the Kalman filtering approach adopted in the present paper, and way more costly 
than an EKF computationally speaking.

\section{PROPOSED INVARIANT EKF}

In the present section we propose a novel extended Kalman filter for the tracking problem (5). Since the state space is not a Lie group, the invariant EKF theory cannot be readily applied. It is however easy to generalize to this problem, as we explain in the following paragraph.

\section{A. Extending the Invariant EKF methodology}

The early theory of the Invariant extended Kalman filter [10], [9] was concerned with invariant dynamics on Lie groups. In [11], the theory was generalized to account for a broader class of dynamical systems on Lie groups and convergence properties were obtained. Indeed, it suffices that the dynamics, defined on a matrix Lie group $G \subset \mathbb{R}^{N \times N}$ with state $\chi_{t} \in G$ and input $u_{t} \in U \subset \mathbb{R}^{l}$, satisfy:

$$
\frac{d}{d t} \chi_{t}=f_{u_{t}}\left(\chi_{t}\right)+\chi_{t} w_{t}
$$

where $w_{t}$ is a continuous white noise, with additional condition

$$
f_{u}(a b)=a f_{u}(b)+f_{u}(a) b-a f-u(I d) b
$$

for all $(u, a, b) \in U \times G \times G$. As early noticed in [21], when the state space consists of a Lie group element $\chi_{t}$ and some additional vector $z_{t} \in \mathbb{R}^{k}$, and the dynamics write

$$
\begin{aligned}
\frac{d}{d t} \chi_{t} & =f_{u_{t}}\left(\chi_{t}, z_{t}\right)+\chi_{t} w_{t}^{\chi}, \\
\frac{d}{d t} z_{t} & =g\left(z_{t}\right)+w_{t}^{z},
\end{aligned}
$$

an Invariant EKF can still be devised formally by merely using an invariant error for the Lie group variable $\chi_{t}$ and a usual linear error for the vector variable $z_{t}$. This comes at a price, though, as convergence properties are lost. However, the structure of the filter which embraces the partial Lie group structure of the state space is relevant, and in practice very good performances can be expected.

Finally note that, a trivial Lie group structure can be defined on such an extended state space, by multiplying the $\chi$ 's and adding the $z$ 's. This route was recently pursued in [12], to estimate pose and velocities of a body in space from pose measurements. Unfortunately, this trivial extension does not make the overall dynamics for the extended state $\left(\chi_{t}, z_{t}\right)$ satisfy condition (9), so convergence properties are lost no matter what, and the end filter is very similar anyway.

\section{B. Proposed filter's structure}

In the present paper we propose to partition the state space using the following Lie group embedding in the special Euclidean group $S E(3)$ using the homogeneous matrices:

$$
\chi_{t}=\left(\begin{array}{cc}
R_{t} & x_{t} \\
0_{1,3} & 1
\end{array}\right), w_{t}=\left(\begin{array}{cc}
\left(w_{t}^{\omega}\right)_{\times} & w_{t}^{x} \\
0_{1,3} & 0
\end{array}\right)
$$

$\chi_{t}$ is an element of $S E(3)$, and letting $z_{t}=\left(\gamma_{t}, \tau_{t}, u_{t}\right)^{T}$, the system (5) can be rewritten in the form (10)-(11), with

$$
f_{\omega, u}:\left(\begin{array}{cc}
R & x \\
0_{1,3} & 1
\end{array}\right) \rightarrow\left(\begin{array}{cc}
R(\omega)_{\times} & R v \\
0_{1,3} & 0
\end{array}\right), \quad g(z)=0_{3,1}
$$

and the observation (6) can be re-written as:

$$
Y_{n}=\chi_{t_{n}}\left(\begin{array}{c}
0_{3,1} \\
1
\end{array}\right)+\left(\begin{array}{c}
V_{n} \\
0
\end{array}\right)
$$

The reader can easily verify condition (9). To linearize the equations and tune the gains, we propose to use a state estimation error of the form $\left(\chi_{t}^{-1} \hat{\chi}_{t}, \hat{z}_{t}-z_{t}\right)$, that is, the error consists of a left-invariant error on $S E(3)$ as concerns the Lie group part of the state space, and a mere difference as concerns the vector part. The error is thus defined as follows:

$$
\eta=\left(\begin{array}{c}
\eta_{t}^{R} \\
\eta_{t}^{x} \\
\eta_{t}^{\gamma} \\
\eta_{t}^{\tau} \\
\eta_{t}^{u}
\end{array}\right)=\left(\begin{array}{c}
R_{t}^{T} \hat{R}_{t} \\
R_{t}^{T}\left(\hat{x}_{t}-x_{t}\right) \\
\hat{\gamma}_{t}-\gamma_{t} \\
\hat{\tau}_{t}-\tau_{t} \\
\hat{u}_{t}-u_{t}
\end{array}\right)
$$

As the conventional EKF, the IEKF relies on two steps. During the propagation step it is a mere copy of the system's equations (5), using the compact notation (10)-(11):

$$
\frac{d}{d t} \hat{\chi}_{t}=f_{\omega_{t}, u_{t}}\left(\hat{\chi}_{t}\right), \frac{d}{d t} \hat{z}_{t}=g\left(\hat{z}_{t}\right)
$$

Whenever a measurement is available, i.e., at $t=t_{n}$, the state is updated

$$
\hat{\chi}_{t_{n}}^{+}=\hat{\chi}_{t_{n}} \exp \left(L_{n}^{\chi}\left(\hat{\chi}_{t_{n}}^{-1} Y_{n}\right)\right), \quad \hat{z}_{t_{n}}^{+}=z_{t_{n}}+L_{n}^{z}\left(\hat{\chi}_{t_{n}}^{-1} Y_{n}\right)
$$

where exp denote the exponential map of $S E(3)$ (see equation (17) for the detailed formula). In other words, the update of $\chi_{t}$ follows directly from [11], whereas the update of $z_{t}$ mimics the one of the conventional EKF. Indeed, the innovation $\hat{\chi}_{t_{n}}^{-1} Y_{n}$ was defined as in the standard IEKF methodology.

Back to the problem's original variables of System (5), the propagation step writes:

$$
\frac{d}{d t} \hat{R}_{t}=\hat{R}_{t}\left(\hat{\omega}_{t}\right)_{\times}, \frac{d}{d t} \hat{x}_{t}=\hat{R}_{t} \hat{v}_{t}, \frac{d}{d t} \hat{\gamma}_{t}=0, \frac{d}{d t} \hat{\tau}_{t}=0, \frac{d}{d t} \hat{v}_{t}=0
$$

where $\hat{\omega}_{t}=\left(\begin{array}{lll}\hat{\tau}_{t} & 0 & \hat{\gamma}_{t}\end{array}\right)^{T}, \hat{v}_{t}=\left(\begin{array}{lll}\hat{u}_{t} & 0 & 0\end{array}\right)^{T}$, and the update writes:

$$
\left(\begin{array}{c}
\hat{R}_{t_{n}}^{+} \\
\hat{x}_{t_{n}}^{+} \\
\hat{\gamma}_{t_{n}}^{+} \\
\hat{\tau}_{t_{n}}^{+} \\
\hat{u}_{t_{n}}^{+}
\end{array}\right)=\left(\begin{array}{c}
\hat{R}_{t_{n}} \exp _{m}\left[\left(\delta_{\omega}\right)_{\times}\right] \\
\hat{R}_{t_{n}} \hat{x}_{t_{n}}+B\left(\delta_{\omega}\right) \delta_{x} \\
\hat{\gamma}_{t_{n}}+\delta_{\gamma} \\
\hat{\tau}_{t_{n}}+\delta_{\tau} \\
\hat{u}_{t_{n}}+\delta_{u}
\end{array}\right)
$$

where $\left(\begin{array}{lllll}\delta_{\omega} & \delta_{x} & \delta_{\gamma} & \delta_{\tau} & \delta_{u}\end{array}\right)^{T}=L_{n}\left(\hat{R}_{t_{n}}^{T}\left(Y_{n}-\hat{x}_{t_{n}}\right)\right), \quad \exp _{m}$ denotes the matrix exponential map in $\mathbb{R}^{3 \times 3}$, and

$$
B(\delta \omega)=I_{3}+\frac{1-\cos \left\|\delta_{\omega}\right\|}{\left\|\delta_{\omega}\right\|^{2}}\left(\delta_{\omega}\right)_{\times}+\frac{\delta_{\omega}-\sin \left\|\delta_{\omega}\right\|}{\left\|\delta_{\omega}\right\|^{3}}\left[\left(\delta_{\omega}\right)_{\times}\right]^{2} .
$$

The gain matrix $L_{n} \in \mathbb{R}^{9 \times 3}$ is computed through a Riccati equation based on linearizations around the estimated trajectory, as in the conventional EKF theory. 


\section{Linearization of the error system and gain computation}

To tune the gains, we need to compute how the error (13) evolves during the propagation, and to write the update step, up to first order terms in the error (linearization). As in the standard EKF, the linear Kalman theory will then allow to estimate the error and its dispersion (covariance matrix) through a Riccati equation.

1) Propagation step: During the propagation step, the system's equations are (5), and the filters' are (16). As a result, the time derivative of the error (13) is

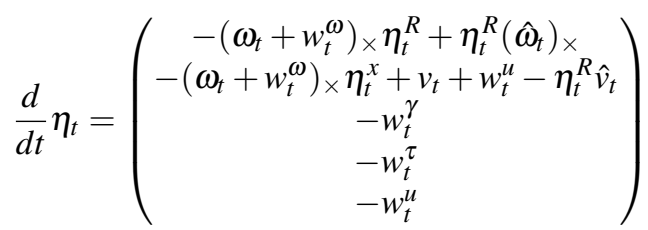

To linearize this equation, following the IEKF methodology, see [11], we let $\eta_{t}^{R} \approx I_{3}+\left(\xi_{t}^{R}\right)_{\times}$. This means that $\xi_{t}^{R} \in \mathbb{R}^{3}$ is a small instantaneous rotation (random Gaussian) vector that encodes the discrepancy in orientation between the true and the estimated vehicles. We also let $\xi_{t}^{x}=\eta_{t}^{x}, \xi_{t}^{\omega}=\eta_{t}^{\omega}$, $\xi_{t}^{\tau}=\eta_{t}^{\tau}, \xi_{t}^{u}=\eta_{t}^{u}$. The rationale, akin to the standard EKF methodology (in the presence of non-additive noise), is then to neglect all second order terms in the components of $\xi$ as well as terms of order $\|\xi\|\|w\|$, that is the noise when it enters multiplicatively the linearized error equation. First we use $\left(\xi_{t}^{R}\right)_{\times}\left(\hat{\omega}_{t}\right)_{\times}-\left(\hat{\omega}_{t}\right)_{\times}\left(\xi_{t}^{R}\right)_{\times}=\left(\xi_{t}^{R} \times \hat{\omega}_{t}\right)_{\times}$. This allows to identify the term $\frac{d}{d t} \xi_{t}^{R}$ we are seeking using that $(a)_{\times}=(b)_{\times}$ implies $a=b$. Let

$$
\xi=\left(\begin{array}{l}
\xi_{t}^{R} \\
\xi_{t}^{x} \\
\xi_{t}^{\gamma} \\
\xi_{t}^{\tau} \\
\xi_{t}^{u}
\end{array}\right) \in \mathbb{R}^{9}
$$

During the propagation step we have $\frac{d}{d t} \xi_{t}=A_{t} \xi_{t}+w_{t}$ with

$$
A_{t}=\left(\begin{array}{ccccccccc}
0 & -\hat{\gamma}_{t} & 0 & 0 & 0 & 0 & 0 & -1 & 0 \\
\hat{\gamma}_{t} & 0 & -\hat{\tau}_{t} & 0 & 0 & 0 & 0 & 0 & 0 \\
0 & \hat{\tau}_{t} & 0 & 0 & 0 & 0 & -1 & 0 & 0 \\
0 & 0 & 0 & 0 & -\hat{\gamma}_{t} & 0 & 0 & 0 & -1 \\
0 & 0 & -\hat{u}_{t} & \hat{\gamma}_{t} & 0 & -\hat{\tau}_{t} & 0 & 0 & 0 \\
0 & \hat{u}_{t} & 0 & 0 & \hat{\tau}_{t} & 0 & 0 & 0 & 0 \\
0 & 0 & 0 & 0 & 0 & 0 & 0 & 0 & 0 \\
0 & 0 & 0 & 0 & 0 & 0 & 0 & 0 & 0 \\
0 & 0 & 0 & 0 & 0 & 0 & 0 & 0 & 0
\end{array}\right)
$$

and with $w_{t}=\left(\left(w_{t}^{\omega}\right)^{T},\left(w_{t}^{x}\right)^{T},\left(w_{t}^{\gamma}\right)^{T},\left(w_{t}^{\tau}\right)^{T},\left(w_{t}^{u}\right)^{T}\right)^{T}$

2) Update step: Here we must compute the impact of the update step (17) on the linearized error (19). To do so, we first note that the innovation as we define it in (15), that is $\hat{\chi}_{t_{n}}^{-1} Y_{n}=\hat{R}_{t_{n}}^{T}\left(Y_{n}-\hat{x}_{t_{n}}\right)$, verifies

$$
\hat{R}_{t_{n}}^{T}\left(Y_{n}-\hat{x}_{t_{n}}\right)=\hat{R}_{t_{n}}^{T}\left(x_{t_{n}}-\hat{x}_{t_{n}}\right)+\hat{R}_{t_{n}}^{T} V_{n}=-\left(\eta_{t_{n}}^{R}\right)^{-1} \eta_{t_{n}}^{x}+\hat{R}_{t_{n}}^{T} V_{n}
$$

and thus since $\eta_{t}^{x}=\xi_{t}^{x}$ and $\left(\xi_{t}^{R}\right)_{\times} \xi_{t}^{x}$ is second order, we have $\hat{R}_{t_{n}}^{T}\left(Y_{n}-\hat{x}_{t_{n}}\right) \approx-H \xi+\hat{R}_{t_{n}}^{T} V_{n}$ where $H \in \mathbb{R}^{3 \times 9}$ is defined by $H=\left(\begin{array}{lll}0_{3,3} & I_{3} & 0_{3,3}\end{array}\right)$. Looking at (17) and using the fact that the first order approximations of $B$ and $\exp _{m}$ be the identity, we easily derive the effect of the update (17) on the error (13), and its linear approximation in $\xi$, see (19), writes

$$
\left.\xi_{t_{n}}^{+}=\xi_{t_{n}}-L_{n}\left[\begin{array}{lll}
\left(0_{3,3}\right. & I_{3} & 0_{3,3}
\end{array}\right) \xi_{t_{n}}-\hat{R}_{t_{n}}^{T} V_{n}\right]
$$

3) Resulting gain: Let $Q_{t}$ be the trusted covariance of the process noise $w_{t}$ and $N_{n}$ the trusted covariance of the measurement noise $V_{n}$. Considering the linearized system obtained with propagation $\frac{d}{d t} \xi_{t}=A_{t} \xi_{t}+w_{t}$ and update $\xi_{t_{n}}^{+}=$ $\xi_{t_{n}}-L_{n}\left[H \xi_{t_{n}}-\hat{R}_{t_{n}}^{T} V_{n}\right]$ the corresponding Kalman gain is obtained through the standard following Riccati equations:

$$
\begin{aligned}
\frac{d}{d t} P_{t} & =A_{t} P_{t}+P_{t} A_{t}^{T}+Q_{t} \\
S_{n} & =H P_{t_{n}} H^{T}+\hat{R}_{t_{n}}^{T} N_{n} \hat{R}_{t_{n}} \\
L_{n} & =P_{t_{n}} H^{T} S^{-1} \\
P_{t_{n}}^{+} & =\left(I_{9}-L_{n} H\right) P_{t_{n}}
\end{aligned}
$$

\section{Discussion on the filter's expected stability}

Unfortunately, as previously underlined, the obtained EKF does not possess convergence guarantees. However, we have the following result, that indicates some stability properties, at least in practice, should be inherited from the IEKF structure of the filter:

Proposition 1: Consider model (5) with noise turned off, measurements with noise turned off too, and assume that the quantities $u_{t}, \gamma_{t}, \omega_{t}$ are all known, and may be freely varying inside an interval $(\alpha, \beta)$ with $\alpha, \beta>0$. Assume also that the lowest eigenvalues of $Q_{t}$ and $N_{n}$ are lower bounded by some $\varepsilon>0$. Then the IEKF proposed above when reduced to the task of estimating $x_{t}$ and $R_{t}$, locally converges around any trajectory of the system.

Proof: The reduced system can be viewed as a nonholonomic car in $3 \mathrm{D}$, that is, evolving in $S E(3)$ instead of $S E(2)$. The result appears then to be a straightforward extension of the simplified car example of [11].

The result above indicates that if the tangential velocity, curvature, and torsion, were known at all times, and if the IEKF were used as a nonlinear observer for the corresponding tracking problem, then this observer would converge. Of course those quantities are not known, so the result above is only partially satisfying. However, it is reassuring as it would not even be provable for an (M)EKF (see [11] indeed).

\section{Simulations}

In the previous section, we derived the filter equations for the tracking problem. In the present section, we test this filtering method on two different trajectories with motions close to real tracking problems.

\section{A. Aircraft trajectory}

The first trajectory tested has been simulated using a trajectory simulation program designed by the company Thales Air Systems. It is inspired by data from a real fighter flight experiment. The trajectory is presented on Figure 1, along with the noisy Cartesian measurements. The results are presented on Figure 2. In all Figures, the red curve 
corresponds to the estimation and the blue one to the real data. Remember that we had defined $\gamma=u \kappa$ and $\tau=u \tilde{\tau}$. So we have estimated $\gamma$ and $\tau$. Here we directly plot $\kappa=\gamma / u$ and $\tilde{\tau}=\tau / u$, the real curvature and the real torsion. For
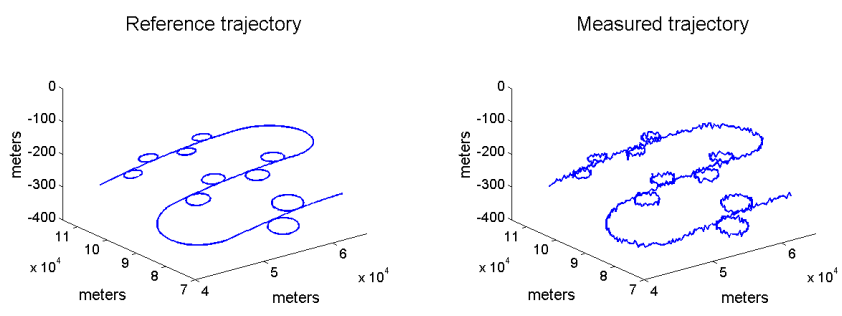

Fig. 1. Reference and noisy aircraft trajectory inspired by real flight data
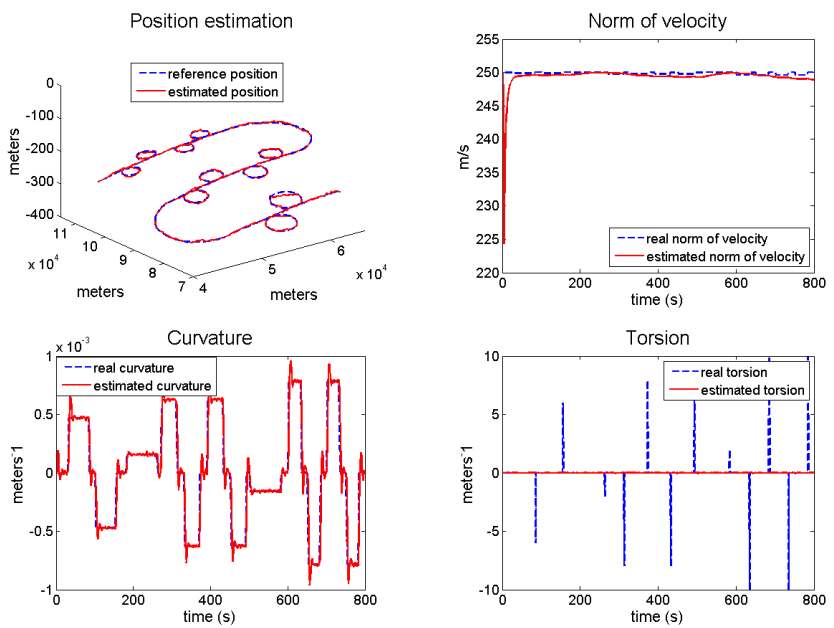

Fig. 2. Estimation of the position (top left) and of parameters $u_{t}$ (top right), $\gamma_{t}$ (bottom left), $\tau_{t}$ (bottom right) for the aircraft trajectory

this trajectory, the position, norm of velocity and curvature are very well estimated by the filter. We see the curvature is in fact piecewise constant, and not nearly constant, but the process noise we added allows the filter to track it very well. The torsion curve shows some peaks. These peaks are filtered out by any algorithm since the quantity measured (the position) is obtained through three integrations of the torsion. More generally, it is hopeless to track the torsion correctly unless it is fairly large and fairly constant over time.

\section{B. Helical trajectory}

To simulate the second trajectory, we describe the evolution of a maneuvering target by (5). We add some measurement Gaussian noise. The noise added is greater along the third coordinate, $\mathrm{z}$ (as it is the case in practice). The reference trajectory and the noisy measured trajectory are presented on Figure 3. We have simulated two consecutive maneuvers. The first one switches from a constant velocity straight line motion to a motion with non-zero curvature and torsion (they remain constant during the motion) along with a change in the norm of the velocity. The second maneuver switches from this last motion to a motion with another constant curvature and norm of velocity and no more torsion. So the target undergoes first a straight line motion, then a helical motion with a change in speed, and then a circle in a plane, with again a change of speed. Such trajectories can be encountered in current air defense applications, and are very challenging to track.
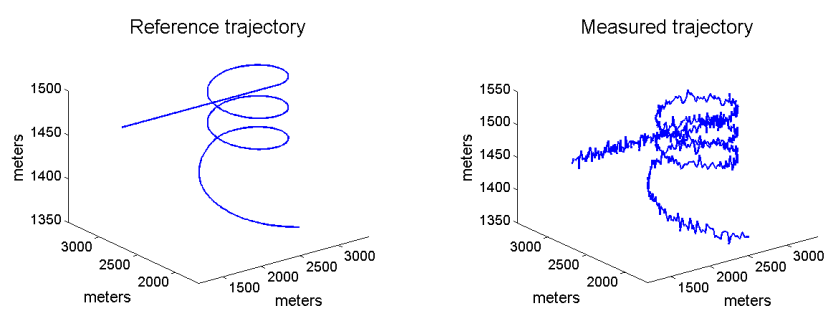

Fig. 3. Reference and noisy trajectory simulated.

We then perform filtering with the IEKF presented in the previous section. The estimations of the position $x$, of the curvature $\kappa$, of the torsion $\tilde{\tau}$, and of the norm of the velocity $u$ are displayed on Figure 4 . We have poorly initialized all parameters to see the behaviour of the filter when confronted to high initial errors (in practice, the position is relatively well-known, but neither are the velocity nor the curvature).
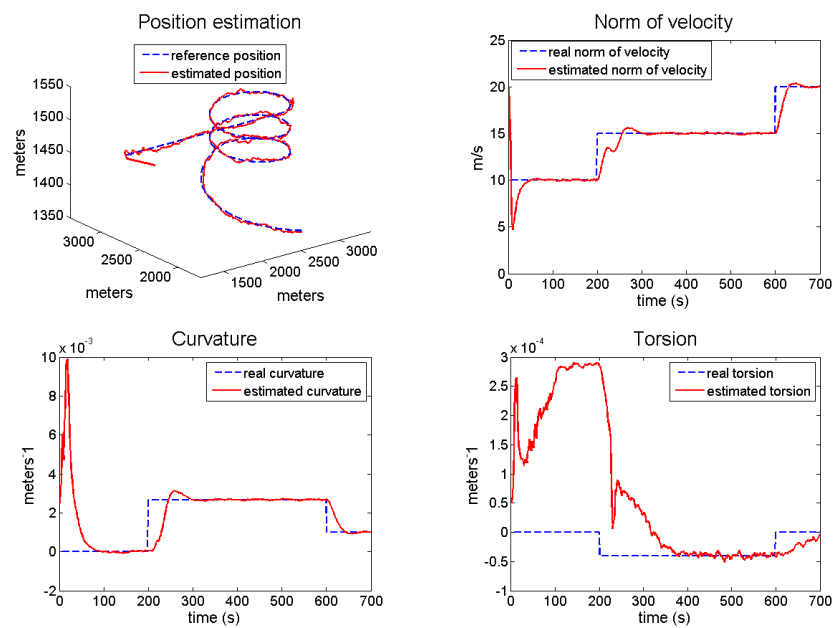

Fig. 4. Estimation of the position (top left) and of parameters $u_{t}$ (top right), $\gamma_{t}$ (bottom left), $\tau_{t}$ (bottom right)

The norm of the velocity and the position are the parts of the state that converge the fastest. The curvature is also quite well estimated, even though it converges slower than the norm of the velocity. The torsion is again the most difficult parameter to estimate, although it eventually converges, it is not well estimated by the filter. This stems from the fact that it is weakly observable since it is small. This is problematic, as even very small values of the torsion lead non negligible helical motions, due to the three integrations over time. However, if it is relatively well-known initially, then the initial covariance can be adjusted so that the initial 
TABLE I

COMPARISON OF THE RMSE FOR THE POSITION AND THE VELOCITY FOR THE IEKF AND CASTELLA'S KALMAN FILTER

\begin{tabular}{|c|c|c|}
\hline Parameter & IEKF & Castella \\
\hline $\mathrm{x}$ & 3.15 & 4.94 \\
\hline $\mathrm{y}$ & 3.15 & 4.91 \\
\hline $\mathrm{z}$ & 5.42 & 2.84 \\
\hline$\dot{\mathrm{x}}$ & 0.76 & 1.51 \\
\hline$\dot{\mathrm{y}}$ & 0.71 & 1.47 \\
\hline$\dot{\mathrm{z}}$ & 0.49 & 0.50 \\
\hline
\end{tabular}

part suits more to the real data, after a jump, it also converges to the true value, if we wait for a sufficiently long time.

\section{Comparison with other filtering algorithms}

As explained in Section II, few filtering algorithms perform well on this kind of trajectories. Indeed, most of the time the models are expressed in two dimensions. The wellknown IMM designed by Bar-Shalom in [1] is itself in 2D, so is the one presented in [22]. It is also difficult to build an Extended Kalman Filter from the Frenet-Serret equations above, because there are orthogonality constraints that cannot easily be dealt with the standard EKF formulation.

The Variable Rate Particle Filter, introduced in [8] might work well, but it involves high computation cost, and the comparison thus seems unfair.

In industrial radars, a well-known trick is to use a linear model (constant acceleration) and adapt the process noise as in the work of Castella [3]. Just to have an idea of what it might yield we have compared the root mean square errors (RMSE) of both filters in Table I. The noises have been tuned manually to get the best possible results with both filters. The Castella seems to perform better for the third coordinate $\mathrm{z}$. It is due to the fact that the noise along this coordinate is higher, and the estimations of the Castella tend to follow the measurement and do not filter the noises enough, especially when it is high.

\section{Conclusions}

We have considered a tracking problem, modeled the trajectory assuming constant norm of the velocity, curvature, and torsion, and cast the model into a Lie group setting. Then, the theory of the invariant EKF, originally devoted to pure Lie groups was adapted to allow the estimation of some extra parameters, namely the norm of the velocity, and the curvature and torsion.

Two challenging tracking examples, close to real industrial problems, have been simulated. The position, the curvature, and the norm of velocity are very well tracked by the filter although they undergo various jumps over the experiment. This is satisfying, as the position and velocity vectors are the only two quantities that need be output with great accuracy. In future work, we plan to integrate our IEKF as a building brick into an IMM or a Rao-Blackwellized particle filter to accommodate jumps and multi-model estimation.

\section{ACKNOWLEDGEMENTS}

This work is partly supported by a DGA-MRIS scholarship and THALES AIR SYSTEMS.

\section{REFERENCES}

[1] Y. Bar-Shalom, X. Li, and T. Kirubarajan, Estimation with Applications to Tracking and Navigation: Theory Algorithms and Software. Wiley, 2004. [Online]. Available: https://books.google.fr/books?id=xz9nQ4wdXG4C

[2] R. A. Singer, "Estimating optimal tracking filter performance for manned maneuvering targets," IEEE Transactions on Aerospace and Electronic Systems, no. 4, pp. 473-483, 1970.

[3] F. Castella, "An adaptive two-dimensional Kalman tracking filter," IEEE Transactions on Aerospace and Electronic Systems, vol. AES16, no. 6, pp. 822-829, Nov. 1980.

[4] F. Daum, "Nonlinear filters: beyond the kalman filter," IEEE Aerospace and Electronic Systems Magazine, vol. 20, no. 8, pp. 57-69, 2005.

[5] R. Mahler, "Phd filters of higher order in target number," IEEE Transactions on Aerospace and Electronic Systems, vol. 43, no. 4, 2007

[6] T. Bullock and S. Sangsuk-Iam, "Maneuver detection and tracking with a nonlinear target model," in Decision and Control, 1984. The 23rd IEEE Conference on, vol. 23. IEEE, 1984, pp. 1122-1126.

[7] R. Bishop and A. Antoulas, "Nonlinear approach to aircraft tracking problem," Journal of Guidance, Control, and Dynamics, vol. 17, no. 5, pp. 1124-1130, 1994.

[8] P. Bunch and S. Godsill, "Dynamical models for tracking with the variable rate particle filter," in Information Fusion (FUSION), 2012 15th International Conference on. IEEE, 2012, pp. 1769-1775.

[9] S. Bonnabel, P. Martin, and E. Salaün, "Invariant extended Kalman filter: theory and application to a velocity-aided attitude estimation problem," in Decision and Control, 2009 held jointly with the 2009 28th Chinese Control Conference. CDC/CCC 2009. Proceedings of the 48th IEEE Conference on. IEEE, 2009, pp. 1297-1304.

[10] S. Bonnabel, "Left-invariant extended kalman filter and attitude estimation," in Decision and Control, 46th IEEE Conference on. IEEE, 2007, pp. 1027-1032.

[11] A. Barrau and S. Bonnabel, "The invariant extended kalman filter as a stable observer," IEEE Transactions on Automatic Control, 2017.

[12] G. Bourmaud, R. Mégret, M. Arnaudon, and A. Giremus, "Continuous-discrete extended Kalman filter on matrix lie groups using concentrated gaussian distributions," Journal of Mathematical Imaging and Vision, vol. 51, no. 1, pp. 209-228, 2014.

[13] P. Martin and E. Salaün, "Generalized multiplicative extended kalman filter for aided attitude and heading reference system," in AIAA Guidance, Navigation, and Control Conference, 2010, p. 8300.

[14] J. R. Forbes, A. H. de Ruiter, and D. E. Zlotnik, "Continuous-time norm-constrained kalman filtering," Automatica, vol. 50, no. 10, pp. 2546-2554, 2014

[15] S. Godsill and J. Vermaak, "Variable rate particle filters for tracking applications," in IEEE/SP 13th Workshop on Statistical Signal Processing, 2005. IEEE, 2005, pp. 1280-1285.

[16] M. Longbin, S. Xiaoquan, Z. Yiyu, S. Z. Kang, and Y. Bar-Shalom, "Unbiased converted measurements for tracking," IEEE Transactions on Aerospace and Electronic Systems, vol. 34, no. 3, pp. 1023-1027, 1998.

[17] N. Nabaa and R. H. Bishop, "Validation and comparison of coordinated turn aircraft maneuver models," IEEE Transactions on aerospace and electronic systems, vol. 36, no. 1, pp. 250-259, 2000.

[18] D. G. Luenberger, "Observing the state of a linear system," IEEE transactions on military electronics, vol. 8, no. 2, pp. 74-80, 1964.

[19] D. Bestle and M. Zeitz, "Canonical form observer design for non-linear time-variable systems," International Journal of control, vol. 38, no. 2 , pp. 419-431, 1983.

[20] Y. Bar-Shalom, K. Chang, and H. A. Blom, "Tracking a maneuvering target using input estimation versus the interacting multiple model algorithm," Aerospace and Electronic Systems, IEEE Transactions on, vol. 25 , no. 2, pp. 296-300, 1989.

[21] M. Barczyk and A. F. Lynch, "Invariant observer design for a helicopter UAV aided inertial navigation system," IEEE Transactions on Control Systems Technology, vol. 21, no. 3, pp. 791-806, 2013.

[22] Y. Boers and J. Driessen, "Interacting multiple model particle filter," IEE Proceedings-Radar, Sonar and Navigation, vol. 150, no. 5, pp. 344-349, 2003 